\title{
Transient Multiple Removal and Subsequent Reimplantation of Abdominal and Retroperitoneal organs with Multisystemic Neoplastic Lesions
}

DOI: $10.17691 / \mathrm{stm} 2017.9 .4 .09$

Received May 3, 2017

A.N. Shcherbyuk, MD, DSc, Professor, Department of Operative Surgery and Topographic Anatomy';

S.S. Dydykin, MD, DSc, Professor, Head of the Department of Operative Surgery and Topographic Anatomy';

K.A. Zhandarov, MD, PhD, Assistant Professor, Department of Operative Surgery and Topographic Anatomy';

V.A. Gulyaev, MD, PhD, Leading Researcher, Department of Kidney and Pancreas Transplantation;

M.H. Bibalaev, Head of the Hospital Admission Unit3;

M.Y. Kapitonova, MD, DSc, Professor of Anatomy, Department of Basic Medical Sciences ${ }^{4}$

1.M. Sechenov First Moscow State Medical University, 8/2 Trubetskaya St., Moscow, 119991, Russian Federation;

2N.V. Sklifosovsky Research Institute of Emergency Care, Moscow City Health Department,

$3 / 21$ Bolshaya Sukharevskaya Sq., Moscow, 129090, Russian Federation;

${ }^{3}$ Pushkino District Hospital named after Prof. V.N. Rozanova, 35 Aviation St., Pushkino, 141200, Moscow Region,

Russian Federation;

${ }^{4}$ Universiti Malaysia Sarawak (UNIMAS), Faculty of Medicine and Health Sciences, Kota Samarahan, Sarawak, Malaysia

The aim of the study was to develop simple, minimally invasive method of transient multiple removal and subsequent reimplantation of abdominal and retroperitoneal organs with multisystemic neoplastic lesions, allowing subsequent reimplantation of the organ block after extracorporeal surgery.

Material and Methods. Ten human cadavers of subjects who died of causes unrelated to pathology of the abdominal organs were used in this study.

The anatomical experiment involved transient multiple removal of the abdominal and retroperitoneal organs with multisystemic neoplastic lesions and their subsequent reimplantation. Surgery was performed using the method developed at the Department of Operative Surgery and Topographic Anatomy of I.M. Sechenov First Moscow State Medical University (Patent RU 2601100).

Results. The proposed method showed several advantages: consistency of cold perfusion in deep hypothermia of the patient, hypothermic circulatory arrest of the abdominal organs (up to 4-6 h); feasibility of insertion and subsequent removal of arterial and venous shunts, reimplantation of the abdominal organs; restoration of arterial and venous blood flow in the patient (cadaver) and preserved integrity of the transected anatomical structures.

Conclusion. The proposed method of transient multiple removal and subsequent reimplantation of the abdominal and retroperitoneal organs with multisystemic neoplastic lesions provides sustained viability of the organs and can be recommended as a suitable model for further application in clinical practice.

Key words: transient multiple removal of the abdominal and retroperitoneal organs; reimplantation of the abdominal and retroperitoneal organs; multisystemic neoplastic lesions; extracorporeal surgery.

Treatment of severe multisystemic diseases of abdominal and retroperitoneal organs with multi-organ neoplastic lesions is a complicated problem of modern surgery which still remains unsolved [1-4].

The available literature provides no description of performing multiple autotransplantation in a clinical setting. There are only a few references regarding surgical treatment of the target organ during its autotransplantation [5].

The aim of the study was to develop simple, minimally invasive method of transient multiple removal and subsequent reimplantation of the abdominal and retroperitoneal organs affected by multisystemic neoplastic lesions, allowing subsequent reimplantation of the organ block after extracorporeal surgery.

Materials and Methods. The method of transient multiple removal and subsequent reimplantation of the abdominal and retroperitoneal organs affected by multisystemic neoplastic lesions [6] was developed on the basis of multiple organ retrieval for transplantation [7] with due consideration of the disadvantages of the latter.

Ten cadavers of the subjects who died from causes unrelated to pathology of the abdominal organs were used in this study.

For contacts: Kirill A. Zhandarov, e-mail: Kirill-zhandarov@mail.ru 
The research was carried out at the mortuary of the N.V. Sklifosovsky Research Institute of Emergency Medicine and approved by the Ethics Committee of I.M. Sechenov First Moscow State Medical University. The study was carried out according to Decree of the Government of the Russian Federation No.750 dated 21 July 2012.

Transient multiple removal and subsequent reimplantation of organs were performed as follows. The abdominal cavity of the cadaver was opened with a midline incision from the xiphoid process to the pubic symphysis. Temporary axillary-femoral arterial and venous shunting were performed. The organ block with the abdominal aorta and the inferior vena cava being removed while maintaining the ability to easily remove the shunts. Simultaneously, we performed clamping of the abdominal aorta below the diaphragm above its bifurcation and of the inferior vena cava above the diaphragm and proximal to the confluence of the common iliac veins and cannulation of these segments of the abdominal aorta and inferior vena cava. Perfusion of the abdominal organs with chilled normal saline or Custodiol solution for up to 4-6 h was done (this allowed to perform surgical treatment of organs with varying degrees of warm ischemic tolerance).

Next, the cardiac part of the stomach, sigmoid colon and ureters were transected. The whole organ block of the abdominal cavity was removed by evisceration and placed on the operating table with corresponding orientation, anterior or retroperitoneal, to form a wide access to the organs. Perfusion with chilled preservative solution was continued. The procedure requires at least 35-40 L of the solution.

Surgical treatment of target organs with neoplastic lesions was simulated. Then the temporary shunts were removed, reimplantation of the abdominal organs was performed, arterial and venous blood flow, as well as integrity of the transected anatomical structures, were restored.

Results. Below there is an example of transient multiple removal of abdominal and retroperitoneal organs with multisystemic neoplastic lesions for surgical treatment (tumor removal) and their subsequent reimplantation.

Removal of a giant tumor with invasion to the left kidney, abdominal aorta, and inferior vena cava was performed after biological death of a 48-year-old man had been established, with the cause of death being an acute traumatic brain injury.

The abdominal cavity of the cadaver was opened with a midline incision from the xiphoid process to the pubic symphysis. The aorta was exposed above the celiac trunk and between the crura of the diaphragm, the ligature being applied under it. The marginal lateral incision was made from the diaphragm to the pelvis on the left and then on the right to dissect the parietal peritoneum and separate the descending colon, the left kidney and expose the duodenum together with the pancreatic head and the left adrenal gland.

Next, the ligamentous apparatus of the liver was transected, the suprahepatic inferior vena cava was exposed, the ligature being applied under it. The inferior vena cava was exposed below the entry of both renal veins and the second ligature was applied under it. The veins associated with the azygos vein system were simultaneously ligated. The abdominal aorta was exposed up to the superior mesenteric artery and fixed, the lumbar arteries (4 pairs) were ligated by separate ligatures.

Two sites were selected on the surface of the aorta and the inferior vena cava for their temporary replacement: one above the upper ligature and the other below the lower ligature, for insertion of the upper and the lower cannula of the bypass. Then the ligatures were tightened and tied in sequence. The cannula connected to the container with chilled preservative solution was inserted into the aorta distal to the upper ligature, the lower ligature being tied on both the aorta and the inferior vena cava.

Waste chilled preservative solution with blood was obtained through the discharge tube inserted into the upper suprahepatic inferior vena cava after it had been ligated by proximal ligature.

Detaching the abdominal and retroperitoneal organs block was continued during perfusion with the solution. The stomach was mobilized by transecting it at the level of cardia, the sigmoid colon and ureters being transected. The lumbar arteries with aorta region were identified.

The obtained organ block was removed from the abdominal cavity and placed on the organ section table, the retroperitoneal surface in the upwards position. Perfusion with chilled preservative solution was continued (40 L).

The vessels of the left kidney and adrenal gland, blood vessels branching from the abdominal aorta and the celiac trunk, venous vessels were isolated from the giant tumor.

Since the organ block was placed with retroperitoneal surface in the upwards position, the kidneys with adrenal glands and the pancreas being uncovered by the parietal peritoneum and unobstructed by adjacent organs, especially the intestine, were well visualized and easily achieved.

Upon completion of the surgery (tumor removal), the abdominal organ block was reimplanted, the arterial and venous bypasses were removed, the patient's arterial and venous blood flow and the integrity of the transected anatomical structures were restored.

Similar operations were performed on nine other cadavers, in eight of which the tumor location and size were identical. In one case, a tumor with invasion into the intestine was removed. In all nine cases, surgical treatment (tumor removal) was carried out without significant technical difficulties.

The proposed method of transient multiple removal 
and subsequent reimplantation of organs [6] differs from multiple organ retrieval for transplantation [7] by permanent perfusion of the abdominal organs with chilled physiological solution (up to 4-6 h), the patient (cadaver) undergoing deep hypothermia.

The method makes it possible to insert and later remove axillary-femoral arterial and venous shunts, replant abdominal organs, restore arterial and venous circulation of the patient (cadaver) and integrity of the transected anatomical structures, which is evidenced by the results of testing other non-operated organs (blood flow simulation on the cadaver).

Additionally, it is possible to carry out removal of target organs with varying degrees of warm ischemic tolerance, which provides reliable viability of all abdominal and retroperitoneal organs.

The area of access to the organs is extended and the time of surgery of the target organ is reduced.

Conclusion. The method of transient multiple removal and subsequent reimplantation of abdominal and retroperitoneal organs provides reliable viability of organs and can serve as a feasible model for later use in the clinical setting.

Contributions of the authors to the study. A.N. Shcherbyuk suggested the investigation concept, S.S. Dydykin and V.A. Gulyaev formulated the aim and objectives, M.Y. Kapitonova participated in the formation of the investigation concept. A.N. Shcherbyuk, S.S. Dydykin and V.A. Gulyaev participated in all stages of the experimental research, K.A. Zhandarov was involved at the stage of transient multiple removal of abdominal and retroperitoneal organs, M.H. Bibalaev was involved at the stage of reimplantation.
Study Funding. This study was not supported by any financial sources.

Conflict of Interests. The authors have no conflict of interests to declare.

\section{References}

1. Justinger C., Schlüter C., Oliviera-Frick V., Kopp B., Rubie C., Schilling M.K. Increased growth factor expression after hepatic and pancreatic resection. Oncol Rep 2008; 20(6): 1527-1531.

2. Ong K.H., Huang S.K.-H., Yen C.-S., Tian Y.-F., Sun D.-P. Simultaneous retroperitoneal robotic partial nephrectomy and hepatectomy for synchronous renal-cell carcinoma and hepatocellular carcinoma in a cirrhotic patient. J Endourol Case Rep 2016; 2(1): 215-217, https://doi.org/10.1089/ cren.2016.0096.

3. Sun J., Yang T., Yang Y., Liu W., Song J. Synchronous double primary malignancies of the liver and kidney: a case report. Oncol Lett 2016; 11(3): 2057-2060, https://doi. org/10.3892/ol.2016.4194.

4. Murodov A.I., Kadyrov Z.A. Simultaneous videoendoscopic surgery in comorbid diseases of abdominal organs and retroperitoneal space. Meditsinskiy vestnik Bashkortostana 2017; 12(3): 129-134.

5. Forni E., Meriggi F. Liver autotransplantation: technique and results. Annaly khirurgicheskoy gepatologii 1998; 3(2): 24-29.

6. Filin A.V., Dydykin S.S., Scherbyuk A.N., Gulyaev V.A. Method of transient multiple removal and subsequent reimplantation of abdominal and retroperitoneal organs with multisystemic neoplastic lesions in experiment. Patent RU 2601100. 2017.

7. Brekhov E.I., Gulyaev V.A., Dydykin S.S., Pavlov A.V. Method of multiple removal of abdominal and retroperitoneal organs for transplantation. Patent RU 1819579. 1993. 